\title{
Facial Geometry Estimation Using Photometric Stereo and Profile Views
}

\author{
Gary A. Atkinson, Melvyn L. Smith, Lyndon N. Smith, and Abdul R. Farooq \\ University of West England, Bristol BS16 1QY, UK \\ Gary.Atkinson@uwe.ac.uk \\ www. uwe.ac.uk/cems/research/groups/mvl/index.html
}

\begin{abstract}
This paper presents a novel method for estimating the threedimensional shape of faces, facilitating the possibility of enhanced face recognition. The method involves a combined use of photometric stereo and profile view information. It can be divided into three principal stages: (1) An initial estimate of the face is obtained using four-source highspeed photometric stereo. (2) The profile is determined from a side-view camera. (3) The facial shape estimation is iteratively refined using the profile until an energy functional is minimised. This final stage, which is the most important contribution of the paper, works by continually deforming the shape estimate so that its profile is exact. An energy is then calculated based on the difference between the raw images and synthetic images generated using the new shape estimate. The surface normals are then adjusted according to energy until convergence. Several real face reconstructions are presented and compared to ground truth. The results clearly demonstrate a significant improvement in accuracy compared to standard photometric stereo.
\end{abstract}

\section{Introduction}

Two of the most important areas of computer vision research are face recognition and 3D surface recovery. This paper makes advances in both these areas by presenting a novel method for estimating the shape of faces from two views. Although the exact method that we describe is principally aimed at faces, only minor modifications are necessary for more general object reconstruction.

Two-dimensional face recognition [1] has been studied for many years, has yielded some promising results and has gained a few basic applications. However, the use of 2D data alone is inherently limited due to the fact that a 2D image of a face varies considerably under different conditions. Factors that affect the 2D appearance of a face include illumination, pose, expression, camera attributes and makeup. Of these, only expression affects the $3 \mathrm{D}$ shape of the face.

This paper describes a method for efficiently recovering the $3 \mathrm{D}$ shape of a face using a combination of photometric stereo and profile view information. The starting point is a device based on four-source Lambertian photometric stereo, which gives an initial estimate of the shape from the frontal view. A high speed camera with rapidly switching light sources is used to avoid problems due 
to face motion. The main contributions of this paper are (1) the hardware set-up used for data capture, (2) our inclusion of a face profile capture mechanism, and (3) an algorithm that we propose to optimise the overall $3 \mathrm{D}$ shape of the face.

The profile is obtained using a side-view camera that captures two images in close succession as the person passes by. The profile shape is then extracted from the images using an energy functional based on the difference between the two images in a $4 \mathrm{D}$ colour space. In order to combine the information from the two views, we apply an energy minimisation approach. The proposed method involves iteratively deforming the current estimate of the surface so that its profile matches that recovered from the side-view camera. The resulting field of surface normals is then used to synthesise new images and the difference between these and the raw images forms the energy functional. The minimisation is performed by adopting a field of normals that is intermediate between the initial estimate and the current estimate, as determined by the calculated energy.

There has been a vast amount of past research into shape reconstruction, and faces in particular, by the computer vision community. Amongst the best-known for 3D face recognition [2]3] are laser-triangulation [4], stereo vision [5], and morphable models 6]. Other, more general, methods that are relevant to our work include shape-from-shading [7] and photometric stereo [89]. Shape-fromshading is used by Zhao and Chellappa who exploit facial symmetry in order to reconstruct faces 10 .

One of the most closely related contributions was proposed by Hernández et al. 11], who attempt to recover generic object shapes. In their work, they use varying views and illumination directions and assume a black background. The silhouettes of the objects are then extracted and used to form an initial estimate of the shape based on the visual hull. The estimate is then optimized based on an energy minimisation scheme similar to the one we present here. Another related work was by Nehab et al. 12, who use range data for the initial estimate of the shape and photometric stereo to reduce noise.

The principal advantages of our method are that (1) only inexpensive equipment is required, (2) the device is able to work in ambient illumination, (3) no assumptions are made about the symmetry of the face (which can be useful for face recognition applications [13), (4) all images are captured almost instantaneously (unlike most other photometric stereo methods), and (5) the processing required for reconstruction is very efficient computationally. The primary disadvantages are the need for rapidly flashing lights and the space required for the two cameras and the four light sources.

\section{Image Acquisition and Single-View Processing}

Figure 1 shows a photograph of the device that we have constructed for data acquisition. The person walks towards camera on the back panel from the left. An ultrasound proximity switch detects the presence of the individual and triggers the acquisition procedure. Frontal images are captured using the high speed camera on the back panel with the face illuminated by the four flashguns in sequence. 


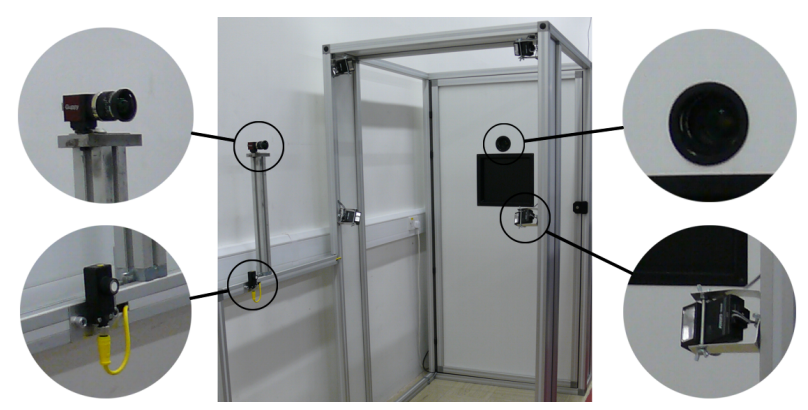

Fig. 1. Photograph of the image capture device. The following features are highlighted: Profile camera (top-left), frontal camera (top-right), ultrasound trigger (bottom-left) and one of the flashguns used in photometric stereo (bottom-right).

Profile images are taken using the camera to the left of Fig. 1 under ambient illumination. The rest of this section details the acquisition and processing of the images and the method used to align the data from each view.

\subsection{Frontal Images}

The first stage of our method is to capture frontal images of the face and make an initial estimate of the 3D geometry using photometric stereo. It is generally expected that the face will be moving at the time of acquisition. For this reason, it is necessary to use a high speed camera to rapidly acquire the images as the light sources change before significant motion is possible. We therefore use a Basler A504kc $1280 \times 1024$ pixel camera operating at $200 \mathrm{fps}$. It was found experimentally that this was the frame rate necessary to avoid face movement between images by more than one pixel. The light sources are Jessops 100M flashguns, which are synchronised to the camera frames. All interfacing and synchronisation is programmed in LabVIEW (although the image processing and shape estimation is performed in MATLAB). The sensor used to initiate the entire process is a highly directional Baumer ultrasound proximity switch.

Four greyscale images are captured by the camera with each corresponding to one of the four light sources shown in Fig. 1. The regions containing the actual face are extracted from the background using the method of Lienhart and Maydt
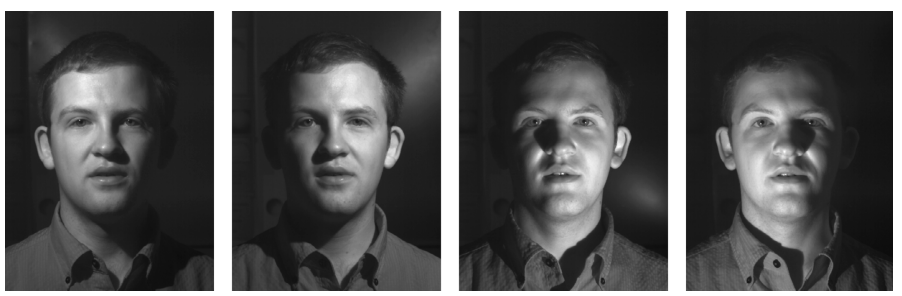

Fig. 2. Example of four raw images, $\mathcal{I}_{1}$ to $\mathcal{I}_{4}$ 
14. This gives us four sets of intensities, $\mathcal{I}_{1}, \mathcal{I}_{2}, \mathcal{I}_{3}$ and $\mathcal{I}_{4}$, each with $N$ pixels, where $\mathcal{I}_{k}=\left\{I_{k, i}, i=1 \ldots N\right\}$. Figure 2 shows an example of four raw images. Notice that, as the lights are bright and the camera exposure is very short, the ambient lighting has negligible impact on the image appearance.

We then calculate an initial estimate of the field of surface normals $\mathcal{V}_{0}=$ $\left\{\mathbf{v}_{0, i}, i=1 \ldots N\right\}$ using a standard photometric stereo technique and assuming known light source directions [15, §5.4]. Finally, we integrate these surface normals using the well-established Frankot-Chellappa method [16] to recover our initial height map estimate, $H_{0}(x, y)$. Figure 3 shows the initial height estimate for the images in Fig. 2 and the definition of the co-ordinate system that we use throughout this paper.

\section{$2.2 \quad$ Profile Images}

In the previous section, we showed how the frontal images were acquired and processed independently of the side-view. In this section we do the opposite and show how to extract the profile shape from the side-view images. The rest of the paper then describes how we combine information from each view.

The side-view camera is shown to the upper-left of Fig. 1. The ultrasound sensor triggers this camera as well as the frontalview camera so that two colour images are captured. These images are taken immediately after the person triggers the device. The camera is an AVT Guppy F-033C $658 \times 494$ camera. The two images are captured in close succession with only a $17 \mathrm{~ms}$ interval between them. Because the person is close to the camera, a wide angle $(4.8 \mathrm{~mm})$ lens was used and a calibration grid employed to correct for distortion. Figure 4 shows an example of a captured side-view image with the detected profile superimposed.

The profile is obtained using the difference between the two images taken $17 \mathrm{~ms}$ apart. In practice, it is insufficient

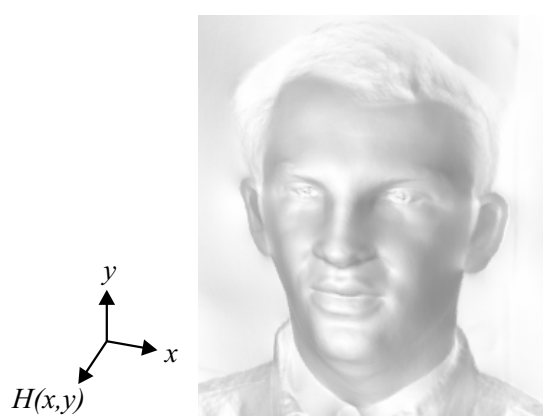

Fig. 3. Height map recovered by applying standard photometric stereo to the images in Fig[2

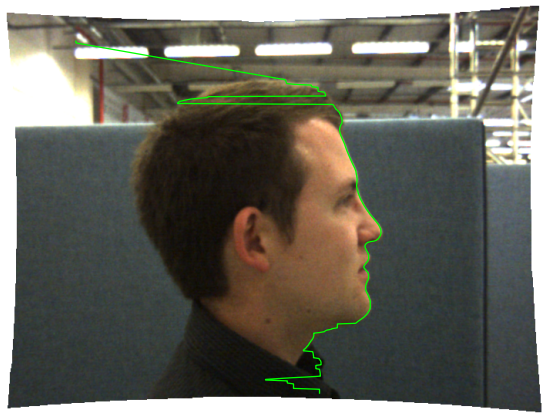

Fig. 4. A side-view image with the detected profile indicated to merely subtract the greylevel intensities since the background may be a similar brightness to the face. Instead, we use an energy functional based on the difference between the red, green and blue components and the colour saturation. The energy for pixel $i$ is then given by: 


$$
E_{P_{i}}=\max \left(\Delta R_{i}, \Delta G_{i}, \Delta B_{i}, \Delta S_{i}\right)
$$

where $\Delta R_{i}, \Delta G_{i}, \Delta B_{i}$ and $\Delta S_{i}$ are the absolute differences between the red, green, blue components and the saturation respectively for the two images. The quantities $R, G, B$ and $S$ are all normalised to fall within the interval [0,1]. We use the maximum differences in (11) as $R, G, B$ and $S$ should all be similar in the background in each image (experiments with the hue yielded no improvement in results). A similar idea to this, using a single image, was proposed by Pantic and Rothkrantz [17.

The profile is extracted from the energy functional defined in (11) by applying:

1. A median smoothing filter to the $E_{P}$ image.

2. Static thresholding to binarise the image, thus segmenting regions of the images that are significantly different from each other.

3. Very basic morphological operations to remove spurious segmented regions.

4. One-dimensional smoothing on the edge of the remaining region of the image to eliminate quantisation noise.

Note that the resulting profile, as shown in Fig. 4, has unsurprisingly been unsuccessful around the hairline. Fortunately, this is not a problem as most face recognition/reconstruction applications are not concerned with hair. Application of our method on a wide range of other faces gave similar results.

\subsection{Data Alignment}

We now have two sets of independently acquired data: the one-dimensional profile, $h(y)$, obtained from the side-view and the height map, $H_{0}(x, y)$, and the field of surface normals, $\mathcal{V}_{0}$, obtained from the frontal view. We now need to align the data from each view and to extract the part of the face useful for recognition (i.e. crop the hair, neck, etc.).

This alignment task is performed with reference to the nose and mouth. First, the location of the nose is detected. In both $H_{0}(x, y)$ and $h(y)$, we assume that this point corresponds to the point of maximum height, i.e. that the subject is facing directly towards

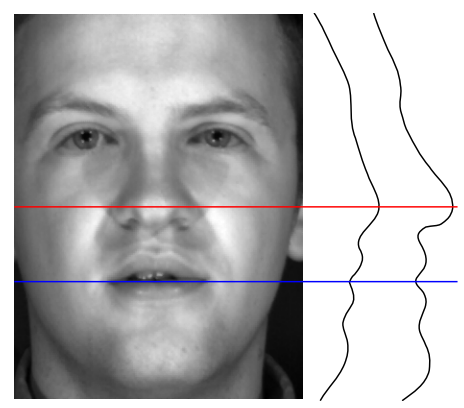

Fig. 5. Image of the cropped region of the face. Profiles are shown taken from $H_{0}(x, y)$ (left) and $h(y)$. The horizontal lines indicate the locations of the nose and mouth. the frontal-view camera. However, in future work we will relax this assumption and by applying a more sophisticated technique [18. The mouth is then found by the second minimum in $H_{0}(x, y)$ and $h(y)$ below the nose (the first minimum being the philtrum).

After locating the nose and mouth, it then becomes a simple task to align the two data sets, as illustrated by Fig. [5. The advantage of using the nose and mouth over, say, the eyes or nasion, is that they are always visible (not occluded 
by hair for example) and can be easily detected in one dimension along the profile.

When the alignment is complete, we extract a fixed fraction of the face images, $\mathcal{I}_{1}$ to $\mathcal{I}_{4}$, the height map, $H_{0}(x, y)$, the surface normals $\mathcal{V}_{0}$, and the profile $h(y)$ ready for the geometrical optimisation described below. The fraction extracted corresponds to half the width and half the height of the raw images (i.e. a quarter of the pixels), centred on the nose location. For simplicity, we shall continue to use the same symbols for the quantities above for the rest of the paper even though they have been truncated in this way. The region of the face detected can be demonstrated by comparing Fig. 5 with Fig. 2.

\section{Geometrical Optimisation}

This section presents the main contribution of the paper. The aim is to deform the estimate of the shape so that its profile matches that of the side-view camera. This must be done in such a way that images synthesised based on the new shape match the raw images as closely as possible. The method that we use is iterative and aims to minimise an energy functional. We use subscripts to indicate the iteration number, where subscript 0 corresponds to the initial estimate.

The following six steps outline how we calculate height estimate $H_{n+1}(x, y)$ from estimate $H_{n}(x, y)$. Further details are given afterwards.

1. Crudely transform the height map $H_{n}(x, y)$ so that its profile matches $h(y)$. Let the result be $H_{n}^{\prime}(x, y)$.

2. Calculate the surface normals of $H_{n}^{\prime}(x, y)$ and call the resulting set of normals $\mathcal{V}_{n}^{\prime}=\left\{\mathbf{v}_{n, i}^{\prime}, i=1 \ldots N\right\}$.

3. Synthesise images based on $\mathcal{V}_{n}^{\prime}$ and the known light source directions.

4. Calculate the energy between the raw image pixels and the synthesised image pixels. This results in a set of energies, $\mathcal{E}=\left\{E_{i}, i=1 \ldots N\right\}$, with each element corresponding to one pixel.

5. Refine the surface normal estimates, $\mathcal{V}_{n}^{\prime}$, based on $\mathcal{E}$ to give the updated normals, $\mathcal{V}_{n+1}$.

6. Calculate the updated height map $H_{n+1}(x, y)$ from $\mathcal{V}_{n+1}$.

For the first step, we adopt a very simple and efficient method. Each horizontal slice of the height map, $H_{n}(x, y)$, is translated in the $z$-direction until the profiles match. Mathematically, for a slice at $y$-position $y_{s}$, the deformed height is given by:

$$
H_{n}^{\prime}\left(x, y_{s}\right)=H_{n}\left(x, y_{s}\right)-\max _{x}\left(H_{n}\left(x, y_{s}\right)\right)+h\left(y_{s}\right)
$$

Figure 6] shows an example result of this operation. The modified set of surface normals, $\mathcal{V}_{n}^{\prime}$, can easily be estimated from $H_{n}\left(x, y_{s}\right)$ by differentiation.

The next task is to synthesise images $\mathcal{I}_{1}^{\prime}$ to $\mathcal{I}_{4}^{\prime}$ corresponding to each of the four light sources. We do this using the standard Lambertian equation:

$$
\mathcal{I}_{k}=\left\{I_{k, i}\right\}=\left\{\rho_{i} \mathbf{l}_{k} \cdot \mathbf{v}_{i}, i=1 \ldots N\right\}
$$


where $\mathbf{l}_{k}$ is the direction vector of the $k^{\text {th }}$ light source and $\rho_{i}$ is the albedo of the $i^{\text {th }}$ pixel estimated using standard photometric stereo. For this paper, we keep the albedo fixed at this value, although in future work we may allow it to vary between iterations.

To complete the fourth step above, an energy functional is needed. Clearly, this will involve the difference between the synthesised intensities, $\mathcal{I}_{1}^{\prime}$ to $\mathcal{I}_{4}^{\prime}$, and the raw images, $\mathcal{I}_{1}$ to $\mathcal{I}_{4}$. We therefore define a pixel-wise set of "intensity energies" by

$$
\mathcal{E}_{I}=\left\{E_{I_{i}}\right\}=\left\{\sum_{k=1}^{4}\left(I_{k, i}-\rho_{i} \mathbf{l}_{k} \cdot \mathbf{v}_{i}\right)^{2}\right\}
$$

However, this is somewhat undesirable for the following reason. We know that the deformed height map, $H_{n}^{\prime}\left(x, y_{s}\right)$ is almost exactly correct at the profile, i.e. along the central vertical line of the face. The region of the face close to the profile should therefore have very low energy. Conversely, far from this line, the estimate is likely to be less accurate. This fact should be represented in the energy functional.

To address the above issue, we introduce a set of confidence indicators, $\mathcal{C}=$ $\left\{C_{i}, i=1 \ldots N\right\}$. It is easiest to define $\mathcal{C}$ initially as a function of $x$ and $y$ as it is based on geometrical considerations. It can then be redefined as a set when being incorporated into the energy functional. The confidence indicator at $(x, y)$ is defined to be proportional to the difference between the height of that point and the height of the profile for position $y$. That is,
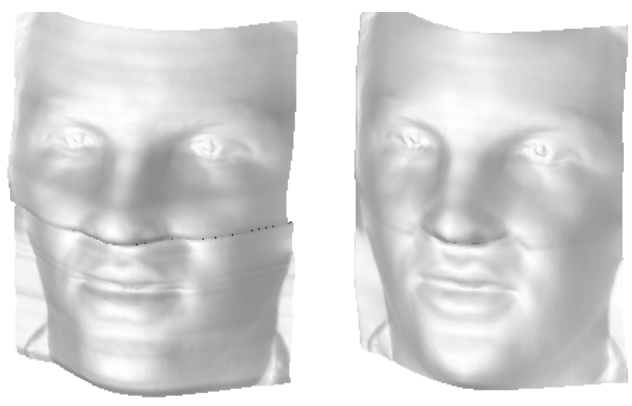

Fig. 6. Left: Result of deforming the initial height map estimate $H_{0}\left(x, y_{s}\right)$ to the match the profile, $h(y)$, resulting in $H_{0}^{\prime}\left(x, y_{s}\right)$. Right: Final estimate of face shape, $H_{M}^{\prime}(x, y)$. At first sight, the final estimate looks very similar to the initial estimate in Fig. 3 . However, the detailed analysis shown in Section 4 shows a significant improvement.

$$
C(x, y)=A\left(h(y)-H_{n}^{\prime}(x, y)\right) \quad \rightarrow \quad \mathcal{C}=\left\{C_{i}\right\}
$$

where $A$ is a parameter which must be optimised experimentally. It turns out that the optimum value that we determined $\left(10^{-5}\right)$ was adequate for all the faces we considered. An adaptive method for selecting the value of $A$ will be the focus of future work.

For regions of the face with low values of $E_{I}$ we would like the surface normals to remain close to the deformed values, $\mathcal{V}_{n}^{\prime}$, whilst for areas of high $E_{I}$, we would like the normals to tend back towards the original surface normal estimates, $\mathcal{V}_{0}$. It is also desirable for areas of high $C$ to follow $\mathcal{V}_{n}^{\prime}$ more closely than for areas of low $C$. We therefore use the following equation to define the total energy: 


$$
\mathcal{E}=\left\{E_{i}\right\}=\left\{\max \left(C_{i} E_{I_{i}}, 1\right)\right\}
$$

where $\mathcal{E}_{I}$ and $\mathcal{C}$ are calculated using (4) and (5) respectively.

The energy now takes values in the interval $[0,1]$. It is then used to modify the surface normals $\mathcal{V}_{n}^{\prime}$ to arrive at the next estimate $\mathcal{V}_{n+1}$. For pixels where $E=0$, then $\mathbf{v}_{n}^{\prime}$ is used as the new surface normal $\mathbf{v}_{n+1}$. Where $E=1$, then $\mathbf{v}_{0}$ is used. Otherwise a surface normal between the two is adopted for the new estimate.

Let the function $R\left(\mathbf{w}_{1}, \mathbf{w}_{2}, \theta\right)$ give a vector found by rotating $\mathbf{w}_{1}$ by an angle of $\theta$ towards $\mathbf{w}_{2}$. The next estimate of surface normal $i$ is then given by the following:

$$
\mathcal{V}_{n+1}=\left\{\mathbf{v}_{n+1, i}\right\}=\left\{R\left(\mathbf{v}_{n, i}^{\prime}, \mathbf{v}_{0, i}, E \arccos \left(\mathbf{v}_{n, i}^{\prime} \cdot \mathbf{v}_{0, i}\right)\right)\right\}
$$

The set of refined surface normals, $\mathcal{V}_{n+1}$ is then re-integrated to form the refined height map $H_{n+1}(x, y)$.

The entire geometrical optimisation process described above is repeated until convergence. If $M$ iterations are required for convergence, then $H_{M}^{\prime}(x, y)$ is used for the final surface. Figure [6] shows an example of this. The iterative procedure takes about four or five seconds to complete on a typical modern personal computer, with cropped face image dimensions of $300 \times 400$ pixels.

\section{Results}

Collectively, Figs. 2 to 6 illustrate the various stages of our proposed algorithm for a typical face. As Fig. 3 demonstrates, standard photometric stereo is able to provide a qualitatively good reconstruction of the face without using any profile view information at all. However, as Fig. 5 shows, the reconstruction is only a very rough estimate of the actual shape. One major strength of our proposed technique is that the profile of the face is almost exactly
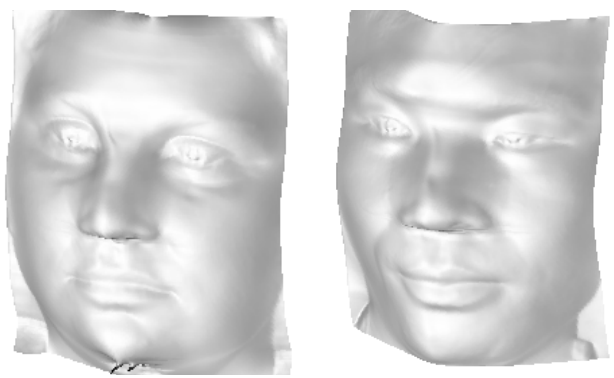

Fig. 7. Surface reconstructions for two of the subjects in Fig. 8] See Fig. 6] for the other reconstruction. correct when the side-view is considered. A slight undesirable consequence of this, is that the nose tends to be widened somewhat. The overall shape estimate is significantly better however for most faces that we considered (and at least a little better for all faces).

Three representative faces are shown in Figs. 6 to 8 . For each face, two vertical cross-sections and two horizontal cross-sections are shown for the initial estimate, $H_{0}(x, y)$, the final estimate, $H_{M}^{\prime}(x, y)$, and ground truth. The ground truth curves were obtained using a $3 \mathrm{DMDface}{ }^{\mathrm{TM}}$ scanner [19]. Note that the righthand vertical cross-sections do not pass through the centre of the face as such profiles match ground truth exactly. 


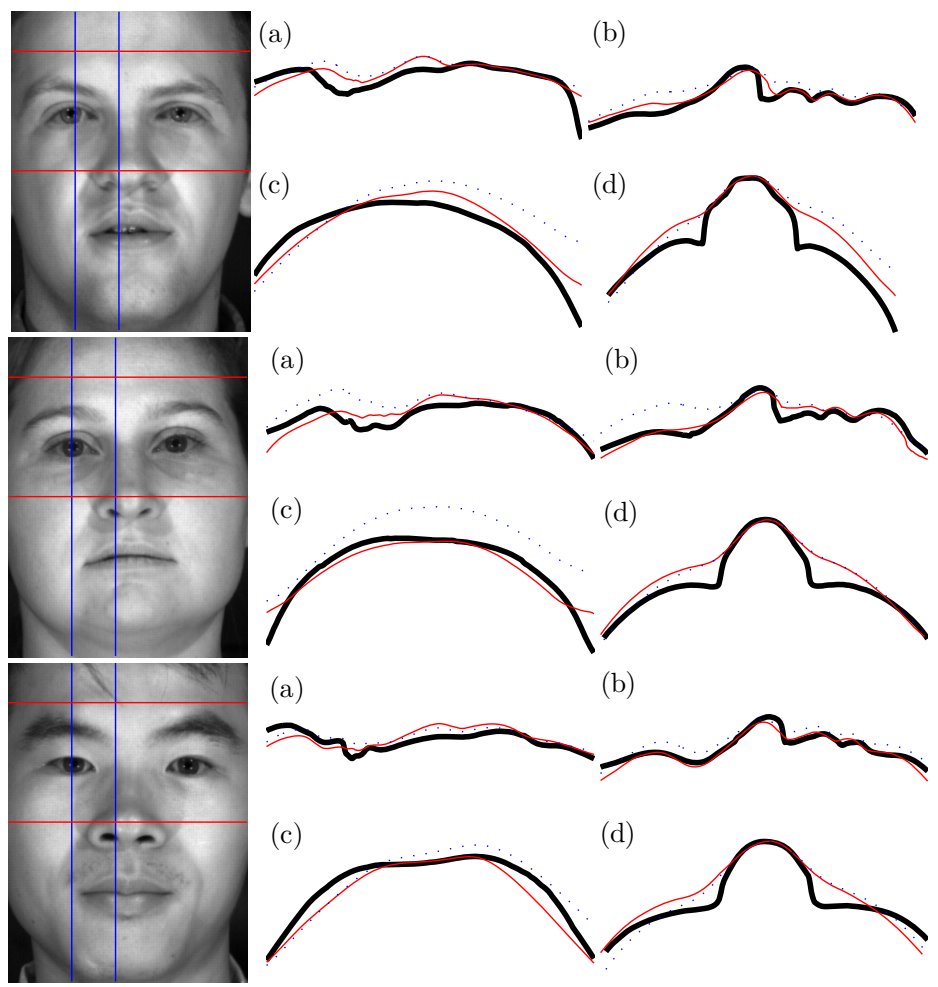

Fig. 8. Cross-sections of face reconstructions for three different subjects. Thick solid lines indicate ground truth, thin solid lines indicate the final estimate and broken lines indicate initial estimates. For each face, curve (a) corresponds to the left vertical line in the image, (b) the right vertical line, (c) the upper horizontal line, and (d) the lower horizontal line.

In all three cases, the final reconstructions match the ground truth reasonably well. In the first two cases, the final estimate is a major improvement over the initial estimate. The horizontal cross-sections across the forehead show particularly impressive results. In order to quantify the improvement to the estimate due the the incorporation of profile information, we have calculated the root-meansquare (RMS) error for the reconstructions when the nose tips are aligned. The reduction in RMS error when the profile information is used is from $26 \mathrm{px}$ to $17 \mathrm{px}$ for the first face, from $23 \mathrm{px}$ to $15 \mathrm{px}$ for the second face, and from $13 \mathrm{px}$ to $12 \mathrm{px}$ for the final face. Note that the small reduction for the final face reflects the fact that the initial estimate was already very good.

\section{Conclusion}

This paper has presented a new method for facial shape estimation using a combination of photometric stereo and profile view information. The paper clearly 
shows a significant improvement in the reconstructions compared to traditional photometric stereo. As shown in Fig. 8, the forehead, the cheeks far away from the nose, and the vertical shape of the nose are all recovered very accurately. This is very useful for face recognition as these areas do not change significantly with expression.

In future work we will aim to allow for more general reflectance properties of the surface. For example, the Lambertian image rendering (3) can be replaced with the Torrance-Sparrow BRDF [20] or other analytic BRDFs. Also, the means by which the energy functional is formed or the way that surface deformation is performed could be improved. Ideally, the deformation should allow for more general shapes to be generated. This should address the problem of the widening noses and possibly allow for spectacles to be reconstructed.

\section{References}

1. Zhao, W., Chellappa, R., Phillips, P.J., Rosenfeld, A.: Face recognition: A literature survey. ACM Computing Surveys 35, 399-458 (2003)

2. Zhao, W., Chellappa, R. (eds.): Face Processing: Advanced Modeling and Methods. Elsevier, Amsterdam (2006)

3. Gupta, S., Markey, M.K., Bovik, A.C.: Advances and challenges in 3D and 2D+3D human face recognition. In: Pattern Recognition Theory and Application. Nova Science Publishers, Inc., New York (2008)

4. Lu, X., Colbry, D.: Matching 2.5D face scans to 3D models. IEEE Trans. Patt. Anal. Mach. Intell. 28, 31-43 (2006)

5. Papatheororou, T., Rueckert, D.: Evaluation of automatic 4D face recognition using surface and texture registration. In: Proc. Automatic Face and Gesture Recognition, pp. 321-326 (2004)

6. Blanz, V., Vetter, T.: A morphable model for the synthesis of 3D faces. In: SIGGRAPH, pp. 87-194 (1999)

7. Zhang, R., Tsai, P.S., Cryer, J.E., Shah, M.: Shape from shading: A survey. IEEE Trans. Patt. Anal. Mach. Intell. 21, 690-706 (1999)

8. Woodham, R.J.: Photometric method for determining surface orientation from multiple images. Optical Engineering 19, 139-144 (1980)

9. Georghiades, A.S., Belhumeur, P.N., Kriegman, D.J.: From few to many: illumination cone models for face recognition under variable lighting and pose. IEEE Trans. Patt. Anal. Mach. Intell. 23, 643-660 (2001)

10. Zhao, W.Y., Chellappa, R.: Symmetric shape-from-shading using self ratio image. Intl. J. Comp. Vis. 45, 55-75 (2001)

11. Hernández, C., Vogiatzis, G., Cipolla, R.: Multiview photometric stereo. IEEE Trans. Patt. Anal. Mach. Intell. 30, 548-554 (2008)

12. Nehab, D., Rusinkiewicz, S., Davis, J., Ramamoorthi, R.: Efficiently combining positions and normals for precise 3D geometry. In: Proc. SIGGRAPH, pp. 536-543 (2005)

13. Liu, Y., Schmidt, K.L., Cohn, J.F., Mitra, S.: Facial asymmetry quantification for expression invariant human identification. Comp. Vis. Im. Understanding 91, 138-159 (2003)

14. Lienhart, R., Maydt, J.: An extended set of Haar-like features for rapid object detection. In: IEEE ICIP, pp. 900-903 (2002) 
15. Forsyth, D.A., Ponce, J.: Computer Vision, A Modern Approach. Prentice-Hall, Upper Saddle River (2003)

16. Frankot, R.T., Chellappa, R.: A method for enforcing integrability in shape from shading algorithms. IEEE Trans. Patt. Anal. Mach. Intell. 10, 439-451 (1988)

17. Pantic, M., Rothkrantz, J.M.: Facial action recognition for facial expression analysis from static face images. IEEE. Trans. Systems, Man and Cybernetics, Part B 34, 1449-1461 (2004)

18. Mian, A.S., Bennamoun, M., Owens, R.: An efficient multimodal 2D-3D hybrid approach to automatic face recognition. IEEE Trans. Patt. Anal. Mach. Intell. 29, 1927-1943 (2007)

19. http://www.3dmd.com/3dmdface.html (January 27, 2009)

20. Torrance, K., Sparrow, M.: Theory for off-specular reflection from roughened surfaces. J. Opt. Soc. Am. 57, 1105-1114 (1967) 